\title{
Differentiable Strategy-Proof Mechanisms for Private and Public Goods in Domains that are not Necessarily Large or Quasi-Linear.*
}

\author{
Luis C. Corchón \\ Departamento de Economía, Universidad Carlos III de Madrid \\ lcorchon@eco.uc3m.es \\ José Rueda-Llano \\ Departamento de Fundamentos del Análisis Económico, \\ Universidad de Alicante \\ joserueda@merlin.fae.ua.es
}

October 10, 2008

\begin{abstract}
In this paper we develop a differentiable approach to deal with incentives in a, possibly small, subset of a general domain of preferences in economies with one public and one private good. We show that, for two agents, there is no mechanism which is efficient, strategy-proof and where consumption of both goods is positive for all agents. For the case of two or more agents the same result occurs when nondictatorship is replaced by Individual Rationality.

Journal of Economic Literature Classification Numbers: D61, D78, H41.

${ }^{*}$ We are very grateful to Atila Abdulkadiroglu, Carmen Beviá, Xavier Calsamiglia, Marco Celentani, Bernardo Moreno, Antonio Romero-Medina, William Thomson, Walter Trockel, an associate editor, three anonymous referees and participants at seminars at Universities of Alicante, Autónoma de Barcelona and Rochester and the Spanish Economic Association meeting in Sevilla for very helpful comments and suggestions.
\end{abstract}




\section{Introduction}

Consider an economy with one public good, one private good and with a finite number of agents each with private information about her preferences. In this framework, a large number of papers have studied the incentives to report information truthfully as a Dominant Strategy, i.e. the so-called strategy-proofness. Most of these papers fall into three strands:

- Papers assuming preferences representable by an utility function linear in the private good, i.e. a Transferrable Utility (TU) domain: Clarke (1971), Groves (1973), Groves and Loeb (1975), Green and Laffont (1977), Laffont and Maskin (1980), Walker (1980), Hurwicz and Walker (1990), Beviá and Corchón (1995) and Schummer (1999).

- Papers assuming that certain, ad hoc, utility functions must belong to the domain: Ledyard and Roberts (1975) for the two agents case, and Saijo (1991) for the $n$ person case.

- Papers assuming that the domain of possible preferences is the set of all continuous, strictly increasing, and strictly quasi-concave utility functions: Serizawa (1996, see pp. 503-8), building on Moulin (1994), characterized mechanisms that are individually rational, no exploitative, and non-bossy. ${ }^{1}$ Variations of this result keeping the assumption on the domain were obtained by Osheto (1997, see p. 160), Deb and Ohseto (1999, see p. 686) and Serizawa (1999, see p. 124).

All these results find that truthful revelation, efficiency and nondictatorship/individual rationality are incompatible requirements. ${ }^{2}$ It is not clear that these results survive when applied to any possible domain of preferences. For instance in a suitably restricted TU domain with more than two agents, truthful revelation, efficiency and non-dictatorship are all compatible: Groves and Loeb (1975) and Tian (1996).

In this paper we consider the incentives problem in a domain of preferences with as little structure as possible. Our main concern is to verify whether the results reviewed above hold in this domain.

\footnotetext{
${ }^{1} \mathrm{~A}$ mechanism is no exploitive if no agent receives a consumption of the private good larger than her endowment. A mechanism is Non-Bossy if no agent can affect the bundle consumed by any other agent without affecting the bundle consumed by her.

${ }^{2}$ Saijo (1991) does not assume efficiency but assumes a large domain and a strong individual rationality requirement.
} 
The problem is that the tools developed so far cannot deal with this goal: In TU domains the efficient quantity of the public good is determined independently of the consumption of the private good which is not generally true in other domains. The approach of Ledyard and Roberts (1975) and Saijo (1991) rely on the construction of specific utility functions. Finally the characterization approach only works in a very large domain of preferences.

We approach this problem by assuming that the relevant functions are continuously differentiable. Thus, first order conditions of payoff maximization gives us information about how the mechanism looks like. Sometimes this information is enough to obtain a result (as in our Proposition 2), sometimes we have to borrow results from the theory of partial differential equations (as in our Proposition 1). We notice that continuous differentiability is just a little bit stronger than continuity because continuous functions can be approximated by differentiable functions, Bartle (1976), p. 172. And continuity is a sensible requirement in mechanism design, Postlewaite and Wettstein (1989) and Peleg (1996). However the differentiable approach eliminates strategy-proof solutions that are not differentiable like the generalized medians and the uniform rule. In any case, it would be interesting to know if the results obtained here hold under non differentiability. This task is beyond the objective of this paper.

Differentiability has been already used in the literature: Laffont and Maskin (1980), (1982) and Satterthwaite and Sonnenschein (1981). The latter is the closest paper to ours. Their main result, recorded as Theorem 1, says that any Non-Bossy, strategy-proof mechanism in which second order conditions of payoff maximization hold with strict inequality (Regularity) and satisfying certain technical properties yields serial dictatorship. We will discuss the relationship of this result with ours' later on.

In Proposition 1 we study the case of two agents. This case is important because many contracts take place between two parties. We show that if the mechanism yields strictly positive consumptions for both agents, efficiency and strategy-proofness are incompatible in any domain in which preferences are representable by an utility function that is additively separable between the public and the private good and in which the marginal rate of substitution between both goods is strictly monotonic on the type of the agent, i.e. the so called single-crossing condition. Our assumption that the mechanism picks up strictly positive consumptions implies that the mechanism is nondictatorial. In certain cases, both properties are equivalent (see Footnote $6)$. 
When the number of agents is more than two, as noticed above, in restricted domains, truthful revelation, efficiency and non-dictatorship are all compatible. Thus, perhaps there are mechanisms fulfilling stronger requirements. A natural step in economies where the property of resources is private and agents have the option to opt out is to ask that the mechanism yields allocations that are weakly preferred to the initial endowments, i.e. individually rationality. Our Proposition 2 says that with two or more agents, efficiency, strategy-proofness, individual rationality and a regularity assumption (that we call Weak Regularity) are incompatible. The latter assumption implies that, for at least one agent, the single-crossing condition holds locally and second order of payoff maximization hold with strict inequality. In fact, our result is even stronger because we only need to assume that the outcome is efficient for a specific profile of preferences. We stress that the domains of preferences used in this paper include those representable by quasi-linear utility functions. Therefore, our results are also applicable to TU domains.

Our results are logically independent of those by Satterthwaite and Sonnenschein (1981): On the one hand the Non-Bossy assumption is implied by our assumptions in Proposition 1 (two agents plus budget balance) and their set up is more general than our's. Finally, they do not assume additively separable utility functions as in our Proposition 1. On the other hand, our assumptions on the domain are weaker, for instance our single-crossing condition is implied by their Regularity condition (see our comments after Remark 1). Our Proposition 2 does not need the Non-Bossy assumption. Finally, we dispose of the additional technical conditions used by these authors that, sometimes, are difficult to interpret.

The rest of the paper goes as follows. Section 2 presents the model and some definitions. Section 3 presents our main results. Section 4 concludes.

\section{The Model}

There are $I$ agents. Let $y \in \mathbb{R}_{+}$be the amount of the public good and $x_{i} \in \mathbb{R}_{+}$the consumption of the private good (money). Let $\omega_{i} \in \mathbb{R}_{++}$be the agent $i$ initial endowment of the private good and $\omega$ be the aggregate endowment of the private good. Thus, $\omega=\sum_{i} \omega_{i}$. An allocation is a vector $\left(y, x_{1}, \ldots, x_{I}\right)$.

We assume that all the relevant functions are twice continuously differentiable. The preferences of agent $i$ are representable by a utility function 
$U_{i}\left(y, x_{i}, \theta_{i}\right)$ concave in $\left(y, x_{i}\right)$ with

$$
\frac{\partial U_{i}\left(y, x_{i}, \theta_{i}\right)}{\partial y}>0 \text { and } \frac{\partial U_{i}\left(y, x_{i}, \theta_{i}\right)}{\partial x_{i}}>0
$$

and where $\theta_{i}$, the type of agent $i$, is drawn from an interval $\Theta_{i}=\left(\underline{\theta}_{i}, \bar{\theta}_{i}\right) \subset \mathbb{R}$.

In Proposition 1 we consider preferences that are representable by a utility function of the form $U_{i}\left(y, x_{i}, \theta_{i}\right)=H(y)+v_{i}\left(x_{i}, \theta_{i}\right)$, where $H(\cdot)$ and $v_{i}\left(\cdot, \theta_{i}\right)$ are concave and strictly increasing, and $\frac{\partial v_{i}\left(y, x_{i}, \cdot\right)}{\partial x_{i}}$ is strictly monotonic in $\theta_{i}$, for every $i$, i.e. the so-called Spence-Mirrlees single-crossing condition. ${ }^{3}$ This property guarantees that for each agent and for any pair of preferences, her associated indifference curves cross just once. ${ }^{4}$ Notice that this utility function can be written as $U_{i}\left(y, x_{i}, \theta_{i}\right)=F_{i}\left(\theta_{i}\right) f(y)+g_{i}\left(x_{i}, \theta_{i}\right)$ with $F_{i}(\cdot)>0$. Two examples of this class of utility functions are Cobb-Douglas, $U_{i}\left(y, x_{i}, \theta_{i}\right)=y^{\alpha_{i}\left(\theta_{i}\right)} x_{i}^{\beta_{i}\left(\theta_{i}\right)}$, or quasi-linear, $U_{i}\left(y, x_{i}, \theta_{i}\right)=\theta_{i} f(y)+x_{i}$ with $\theta_{i}>0 .^{5}$

A vector $\theta=\left(\theta_{1}, \theta_{2}, \ldots, \theta_{I}\right)$ will be called an economy. The space of all possible economies is denoted by $\Theta=\prod_{i=1}^{I} \Theta_{i}$. The cost function, denoted by $c(\cdot)$, is also twice continuously differentiable with $c^{\prime}(y)>0$, and $c^{\prime \prime}(y) \geq 0$, for every $y$. The set of feasible allocations is

$$
A \equiv\left\{\left(y, x_{1}, \ldots, x_{I}\right): y \in \mathbb{R}_{+}, x_{i} \in \mathbb{R}_{+} \text {for all } i, \sum_{i=1}^{I} x_{i}+c(y) \leq \omega\right\} .
$$

An allocation $\left(y, x_{1}, \ldots, x_{I}\right)$ is Individually Rational in economy $\left(\theta_{1}, \theta_{2}, \ldots, \theta_{I}\right)$ if $U_{i}\left(y, x_{i}, \theta_{i}\right) \geq U_{i}\left(0, \omega_{i}, \theta_{i}\right)$ for all $i$.

A Social Choice Function (SCF) is a function $f: \Theta \rightarrow A$. We will write $f(\cdot)=\left(y(\cdot), x_{1}(\cdot), \ldots, x_{I}(\cdot)\right)$. A SCF is Individually Rational (IR) if for each economy it selects individually rational allocations. This is an appropriate requirement for economies in which the property of the private good is private and agents have the option of not participating in the mechanism. A SCF $f(\cdot)$ is Budget Balanced (BB) if,

$$
\sum_{i=1}^{I} x_{i}(\theta)+c(y(\theta))=\omega, \forall \theta \in \Theta
$$

\footnotetext{
${ }^{3}$ This property ia also used in principal-agent models and signalling. See Sapority (2007) for an application to pure public good economies

${ }^{4}$ In Proposition 2 the single-crossing condition will be derived from our Weak Regularity assumption, see Definition 1 below.

${ }^{5}$ Not every quasi-linear utility function can be written in the way we assumed above.
} 
A SCF $f(\cdot)$ is Pareto Efficient $(\mathrm{PE})$ if there is no economy $\theta=\left(\theta_{1}, \ldots, \theta_{I}\right)$ and feasible allocation $\left(y, x_{1}, \ldots, x_{I}\right) \in A$ such that $U_{i}\left(y, x_{i}, \theta_{i}\right) \geq U_{i}\left(f(\theta), \theta_{i}\right)$ for every $i$ with, at least, one inequality strict. If an allocation is $\mathrm{PE}$ and interior (see Campbell and Truchon (1988) and Conley and Diamantaras (1996) for non-interior PE allocations) it must satisfy the Lindahl-Bowen-Samuelson condition

$$
c^{\prime}(y)=\sum_{i=1}^{I} \frac{\frac{\partial U_{i}\left(y, x_{i}, \theta_{i}\right)}{\partial y}}{\frac{\partial U_{i}\left(y, x_{i}, \theta_{i}\right)}{\partial x_{i}}}
$$

which for $U_{i}\left(y, x_{i}, \theta_{i}\right)=H(y)+v_{i}\left(x_{i}, \theta_{i}\right)$ can be written as

$$
c^{\prime}(y)=\frac{\partial H(y)}{\partial y} \sum_{i=1}^{I} \frac{1}{\frac{\partial v_{i}\left(x_{i}, \theta_{i}\right)}{\partial x_{i}}} .
$$

Notice that when preferences are quasi-linear in money the previous equation determines the level of the public good. However in our framework this is not necessarily the case: For instance, let $I=2, u_{i}=y+F\left(\theta_{i}\right) x_{i}^{\beta}, 0<$ $\beta<1, F\left(\theta_{i}\right)>0$, and $c(y)=y$. Assuming interiority, the Lindahl-BowenSamuelson equation can be written as

$$
x_{2}(\theta)=\left[\frac{\left[\beta F\left(\theta_{1}\right)-x_{1}(\theta)^{1-\beta}\right] F\left(\theta_{2}\right)}{F\left(\theta_{1}\right)}\right]^{\frac{1}{1-\beta}} .
$$

Thus, in this case PE does not determine the level of the public good -as under quasi-linear preferences- but a locus of points $\left(x_{1}, x_{2}\right){ }^{6}$

Let $\Delta$ be the $I-1$ unit simplex and $\operatorname{int}(\Delta)$ be the interior of $\Delta$. Under our assumptions, a SCF $f(\cdot)$ is $\mathrm{PE}$ if there exist $I$ functions $\alpha_{1}(\cdot), \ldots, \alpha_{I}(\cdot) \in \Delta$ with domain $\Theta$ and such that, for every economy $\theta=\left(\theta_{1}, \ldots, \theta_{I}\right)$,

$$
f(\theta) \in \arg \max _{y, x_{1}, \ldots, x_{I}} \sum_{i=1}^{I} \alpha_{i}(\theta)\left[U_{i}\left(y, x_{i}, \theta_{i}\right)\right] \text { with } \sum_{i=1}^{I} x_{i}+c(y)=\omega .
$$

A Pareto efficient SCF is non-dictatorial if $\alpha_{i}(\cdot) \neq 1$, for any $i$.

In this paper we focus on direct mechanisms where the agent $i$ 's set of possible messages (strategies) is $\Theta_{i}$ and the outcome function is the SCF.

\footnotetext{
${ }^{6}$ Keser [14], worked out a special case of the utility functions assumed in this example. She noticed that in this domain, the contribution game has a dominant strategy.
} 
Let $\theta_{-i}=\left(\theta_{1}, \ldots, \theta_{i-1}, \theta_{i+1}, \ldots, \theta_{I}\right), \theta=\left(\theta_{i}, \theta_{-i}\right)$. The $\operatorname{SCF} f(\cdot)$ is dominant strategies incentive compatible (DSIC) (or strategy-proof) if

$$
U_{i}\left(f\left(\theta_{i}, \theta_{-i}\right), \theta_{i}\right) \geq U_{i}\left(f\left(\theta_{i}^{\prime}, \theta_{-i}\right), \theta_{i}\right) \text { for all } i, \theta_{i}, \theta_{i}^{\prime} \in \Theta_{i}, \theta_{-i} \in \Theta_{-i},
$$

where, by abusing notation the function $U_{i}\left(\cdot, \theta_{i}\right)$ includes the whole allocation in its argument.

\section{Results}

In this section we gather our main findings. We assume the following:

Assumption 1. The SCF is $\mathcal{C}^{1}$ with strictly positive values, defined on an open set $\Theta$ in which utility functions can be written as $H(y)+v_{i}\left(x_{i}, \theta_{i}\right)$, where

$H(\cdot)$ and $v_{i}\left(\cdot, \theta_{i}\right)$ are concave and strictly increasing and $\frac{\partial v_{i}\left(y, x_{i}, \cdot\right)}{\partial x_{i}}$ is strictly monotonic in $\theta_{i}$ for every $i$.

Assumption 1 (A.1 in the sequel) says three things. Firstly, that the SCF is continuously differentiable and yields strictly positive consumption of private and public goods for all agents. This implies that the SCF is non-dictatorial, i.e. that $\alpha_{i} \neq 1$, for any $i .^{7}$ This assumption is akin to the "Minimum Consumption Guarantee" in Serizawa and Weymark (2003). Secondly it requires that utility functions are additively separable between private and public goods. Finally it requires that the marginal rate of substitution between the private and the public good is monotonic with the type, i.e. the single-crossing condition.

Let us begin by proving three lemmas which characterize the properties of strategy-proof and efficient SCF satisfying A.1. Lemma 1 works for any number of agents but Lemmas 2 and 3 require two agents.

Lemma 1. Under $A$. 1, if a SCF $f(\cdot)=\left(y(\cdot), x_{1}(\cdot), \ldots, x_{I}(\cdot)\right)$ is PE and $D S I C$, then there exist functions $\alpha_{1}(\cdot), \ldots, \alpha_{I}(\cdot) \in \operatorname{int}(\Delta)$ such that

$$
\alpha_{i}(\theta) \sum_{j=1}^{I} \frac{\partial x_{j}(\theta)}{\partial \theta_{i}}=\frac{\partial x_{i}(\theta)}{\partial \theta_{i}} \text {, for every } i=1, \ldots, I \text { and every } \theta \in \Theta .
$$

${ }^{7}$ When $I=2$ and goods are essential (If $\left(y^{\prime}, x_{i}^{\prime}\right)>>0, U_{i}\left(y^{\prime}, x_{i}^{\prime}\right)>U_{i}\left(y^{\prime \prime}, x_{i}^{\prime \prime}\right)$ for any $\left(y^{\prime \prime}, x_{i}^{\prime \prime}\right)$ with a zero component) it is easy to show that if a SCF is Pareto Efficient and Non-Dictatorial, it must yield strictly positive consumption of both goods to all agents. 
Proof. Since $f(\cdot)$ take strictly positive values we can work with the first order conditions. Since SCF is PE, from the first order conditions of the maximization of $\sum_{i=1}^{I} \alpha_{i}(\theta)\left[H(y)+v_{i}\left(x_{i}, \theta_{i}\right)\right]$ over $\sum_{i=1}^{I} x_{i}+c(y)=\omega$, we have that for every $\theta \in \Theta$, the following equations hold:

$$
\begin{gathered}
\alpha_{i}(\theta) \frac{\partial v_{i}\left(x_{i}(\theta), \theta_{i}\right)}{\partial x_{i}}=\frac{1}{c^{\prime}(y(\theta))} \frac{\partial H(y)}{\partial y}, \text { for every } i=1, \ldots, I, \\
\sum_{i=1}^{I} x_{i}(\theta)+c(y(\theta))=\omega .
\end{gathered}
$$

If the SCF is also DSIC, then, for every $\theta \in \Theta$ and $i$,

$$
\frac{\partial H(y)}{\partial y} \frac{\partial y(\theta)}{\partial \theta_{i}}+\frac{\partial v_{i}\left(x_{i}(\theta), \theta_{i}\right)}{\partial x_{i}} \frac{\partial x_{i}(\theta)}{\partial \theta_{i}}=0 .
$$

Now, by differentiating condition (3) with respect to $\theta_{i}$ we have that, for every $\theta \in \Theta$ and for every $i$,

$$
\sum_{j=1}^{I} \frac{\partial x_{j}(\theta)}{\partial \theta_{i}}+c^{\prime}(y(\theta)) \frac{\partial y(\theta)}{\partial \theta_{i}}=0 .
$$

From conditions (2), (4), and (5) the result follows.

Next two Lemmas deal with the two person case. In order to simplify notation, we will write $\alpha_{1}(\cdot)=\alpha(\cdot)$ and $\alpha_{2}(\cdot)=1-\alpha(\cdot)$.

Lemma 2. Assume A.1 and that $I=2$. Then, the solution of the partial differential equation (1) can be written as a $\mathcal{C}^{1}$ function of $\alpha(\cdot)$.

Proof. Write the system of partial differential equations (1) as follows:

$$
\begin{aligned}
\frac{\partial x_{1}(\theta)}{\partial \theta_{1}} & =\frac{\alpha(\theta)}{1-\alpha(\theta)} \frac{\partial x_{2}(\theta)}{\partial \theta_{1}}, \\
\frac{\partial x_{1}(\theta)}{\partial \theta_{2}} & =\frac{\alpha(\theta)}{1-\alpha(\theta)} \frac{\partial x_{2}(\theta)}{\partial \theta_{2}} .
\end{aligned}
$$

If there exist functions $x_{1}(\cdot)$ and $x_{2}(\cdot)$ that solve the above equations, by differentiating with respect to $\theta_{2}$ in (6) and with respect to $\theta_{1}$ in (7), these 
functions have to satisfy

$$
\begin{aligned}
\frac{\partial^{2} x_{1}(\theta)}{\partial \theta_{1} \theta_{2}} & =\frac{\partial\left[\frac{\alpha(\theta)}{1-\alpha(\theta)}\right]}{\partial \theta_{2}} \frac{\partial x_{2}(\theta)}{\partial \theta_{1}}+\frac{\alpha(\theta)}{1-\alpha(\theta)} \frac{\partial^{2} x_{2}(\theta)}{\partial \theta_{1} \theta_{2}} \\
\frac{\partial^{2} x_{1}(\theta)}{\partial \theta_{2} \theta_{1}} & =\frac{\partial\left[\frac{\alpha(\theta)}{1-\alpha(\theta)}\right]}{\partial \theta_{1}} \frac{\partial x_{2}(\theta)}{\partial \theta_{2}}+\frac{\alpha(\theta)}{1-\alpha(\theta)} \frac{\partial^{2} x_{2}(\theta)}{\partial \theta_{2} \theta_{1}}
\end{aligned}
$$

Since for any twice continuously differentiable functions $x_{1}(\cdot)$ and $x_{2}(\cdot)$,

$$
\frac{\partial^{2} x_{1}(\theta)}{\partial \theta_{1} \theta_{2}}=\frac{\partial^{2} x_{1}(\theta)}{\partial \theta_{2} \theta_{1}} \text { and } \frac{\partial^{2} x_{2}(\theta)}{\partial \theta_{1} \theta_{2}}=\frac{\partial^{2} x_{2}(\theta)}{\partial \theta_{2} \theta_{1}},
$$

we have that

$$
\frac{\partial\left[\frac{\alpha(\theta)}{1-\alpha(\theta)}\right]}{\partial \theta_{2}} \frac{\partial x_{2}(\theta)}{\partial \theta_{1}}-\frac{\partial\left[\frac{\alpha(\theta)}{1-\alpha(\theta)}\right]}{\partial \theta_{1}} \frac{\partial x_{2}(\theta)}{\partial \theta_{2}}=0,
$$

which is a linear partial differential equation in $x_{2}$.

Also, we can write equations (6) and (7), as follows:

$$
\begin{aligned}
\frac{\partial x_{2}(\theta)}{\partial \theta_{1}} & =\frac{1-\alpha(\theta)}{\alpha(\theta)} \frac{\partial x_{1}(\theta)}{\partial \theta_{1}}, \\
\frac{\partial x_{2}(\theta)}{\partial \theta_{2}} & =\frac{1-\alpha(\theta)}{\alpha(\theta)} \frac{\partial x_{1}(\theta)}{\partial \theta_{2}} .
\end{aligned}
$$

Then, by differentiating with respect to $\theta_{2}$ in (9) and with respect to $\theta_{1}$ in (10), and applying an analogous reasoning of symmetry of the second cross derivatives of $x_{1}(\cdot)$ and $x_{2}(\cdot)$, we get

$$
\frac{\partial\left[\frac{1-\alpha(\theta)}{\alpha(\theta)}\right]}{\partial \theta_{2}} \frac{\partial x_{1}(\theta)}{\partial \theta_{1}}-\frac{\partial\left[\frac{1-\alpha(\theta)}{\alpha(\theta)}\right]}{\partial \theta_{1}} \frac{\partial x_{1}(\theta)}{\partial \theta_{2}}=0,
$$

which is a linear partial differential equation in $x_{1}$.

The solution to Equations (8) and (11) is found in Zachmanouglou and Thoe (1986, pp. 62, Example 2.2 and Problem 2.3) and is given by

$$
\begin{aligned}
& x_{1}(\theta)=h_{1}(\alpha(\theta)) \\
& x_{2}(\theta)=h_{2}(\alpha(\theta)),
\end{aligned}
$$


where $h_{i}(\cdot)$ is an arbitrary $\mathcal{C}^{1}$ function of a single variable and $\alpha(\theta)=K$ is the general solution of the ordinary differential equation associated with both Equation (8) and Equation (11).8,9

By Budget Balance we can also write

$$
y(\theta)=g(\alpha(\theta))=K^{-1}\left(\omega-h_{1}(\alpha(\theta))-h_{2}(\alpha(\theta))\right) .
$$

All these establish the result.

Lemma 3. Assume A.1 and that $I=2$. Suppose that $f(\cdot)$ is PE and DSIC $S C F$ with an associated function $\alpha \in \operatorname{int}(\Delta)$. Then, $\frac{\partial \alpha(\theta)}{\partial \theta_{i}} \neq 0, i=1,2$.

Proof. Suppose that $\frac{\partial \alpha(\theta)}{\partial \theta_{i}}=0$, some $i$, say $i=1$. By PE (Equation (2) in Lemma 1) and Lemma 2 we have that $\forall \theta \in \Theta$

$$
\alpha(\theta) \frac{\partial v_{1}\left(h_{1}(\alpha(\theta)), \theta_{1}\right)}{\partial x_{1}}=\frac{1}{c^{\prime}(g(\alpha(\theta)))} \frac{\partial H(g(\alpha(\theta)))}{\partial y} .
$$

Differentiating (2') with respect to $\theta_{1}$ we see that the left hand side is different from zero because of the single-crossing condition, i.e.

$$
\begin{gathered}
\alpha(\theta)\left[\frac{\partial^{2} v_{1}\left(x_{1}, \theta_{1}\right)}{\partial x_{1}^{2}} h_{1}^{\prime}(\alpha(\theta)) \frac{\partial \alpha(\theta)}{\partial \theta_{1}}+\frac{\partial^{2} v_{1}\left(x_{1}, \theta_{1}\right)}{\partial x_{1} \theta_{1}}\right]=\alpha(\theta) \frac{\partial^{2} v_{1}\left(x_{1}, \theta_{1}\right)}{\partial x_{1} \theta_{1}} \neq 0, \\
\text { but since } \frac{\partial \alpha(\theta)}{\partial \theta_{1}}=0 \text { we have that } \frac{\partial\left(\frac{\left.\frac{\partial H(g(\alpha(\theta)))}{\partial y(g(\alpha(\theta)))}\right)}{\partial \theta_{1}}=0 .\right.}{}=0 .
\end{gathered}
$$

Therefore equation ( $\left.2^{\prime}\right)$ does not hold for all $\theta$ and we obtain a contradiction. A similar argument can be done with $\theta_{2}$ and so the proof follows.

With these lemmas in hand we prove the following result.

Proposition 1. Under A.1, when $I=2$ there is no SCF that is PE and $D S I C$.

\footnotetext{
${ }^{8}$ The bridge between the notation in Zachmanouglou and Thoe [28] and ours is: $x=\theta_{1}$, $y=\theta_{2}, a(x, y)=\frac{\partial\left[\frac{\alpha(\theta)}{1-\alpha(\theta)}\right]}{\partial \theta_{2}}, b(x, y)=-\frac{\partial\left[\frac{\alpha(\theta)}{1-\alpha(\theta)}\right]}{\partial \theta_{1}}, z=x_{1}$ in (11) and $z=x_{2}$ in (8).

${ }^{9} \mathrm{By}$ making some algebra, the ordinary differential equation can be written as $\frac{\partial \alpha(\theta)}{\partial \theta_{1}} d \theta_{1}+\frac{\partial \alpha(\theta)}{\partial \theta_{2}} d \theta_{2}=0$ and trivially $\alpha(\theta)=K$ is its general solution.
} 
Proof. Suppose there exists a SCF $f(\cdot)=\left(y(\cdot), x_{1}(\cdot), x_{2}(\cdot)\right)$ that is PE and DSIC in some open set $\Theta$ fulfilling A.1. Then, by Lemmas 1 and 2, this SCF has to be of the form:

$$
x_{1}(\theta)=h_{1}(\alpha(\theta)), x_{2}(\theta)=h_{2}(\alpha(\theta)), y(\theta)=g(\alpha(\theta)) .
$$

By Lemma $3 \frac{\partial \alpha(\theta)}{\partial \theta_{i}} \neq 0, i=1,2$. Now consider a change in both $\theta_{1}$ and $\theta_{2}$ around $\theta$ that leaves $\alpha$ unchanged, i.e.

$$
\frac{d \theta_{2}}{d \theta_{1}}=-\frac{\frac{\partial \alpha(\theta)}{\partial \theta_{1}}}{\frac{\partial \alpha(\theta)}{\partial \theta_{2}}}
$$

But again (applying the same reasoning as in the proof of Lemma 3) the single-crossing condition plus the fact that, although both $\theta_{1}$ and $\theta_{2}$ have changed $\alpha$ has not changed, imply that Equation (2') cannot hold.

As pointed out by Groves and Loeb (1975), PE, non-dictatorship and DSIC are compatible in small domains when $I>2$. In fact, if endowments are large enough and the domain of preferences is chosen adequately, it is easy to see that the mechanism proposed by Tian (1996) yields strictly positive consumptions for all agents. Thus, Proposition 1 fails when the number of agents exceeds two. Thus, in this case we may get more ambitious and ask for SCF's to fulfill even stronger properties.

We first need some extra definitions and notation. A SCF $f$ is Weakly Regular (WR) at $\tilde{\theta}$ if there is an agent, say $i$, for whom to tell the truth implies that the second order conditions of payoff maximization hold with strict inequality. Recall that the first order condition of DSIC is

$$
\frac{\partial U_{i}\left(y(\theta), x_{i}(\theta), \tilde{\theta}_{i}\right)}{\partial y} \frac{\partial y(\theta)}{\partial \theta_{i}}+\frac{\partial U_{i}\left(y(\theta), x_{i}(\theta), \tilde{\theta}_{i}\right)}{\partial x_{i}} \frac{\partial x_{i}(\theta)}{\partial \theta_{i}}=0 \text { for } \theta_{i}=\tilde{\theta}_{i} .
$$

where $\theta$ is the economy announced by agents and $\tilde{\theta}_{i}$ is the true type of $i$. Let the left hand side of $(12)$ be denoted by $\Omega\left(\theta, \tilde{\theta}_{i}\right)$. With this notation in hand, we have the following definition:

Definition 1. $f$ is Weakly Regular (WR) at $\tilde{\theta}$ if $\exists i$, with $\frac{\partial \Omega\left(\theta, \tilde{\theta}_{i}\right)}{\partial \theta_{i}}<0$, for $\theta_{i}=\tilde{\theta}_{i}$.

The WR condition is a much weaker version of an assumption called Regularity (R) by Satterthwaite and Sonnenschein (1981, p. 590). R requires 
that the second order condition of payoff maximization holds strictly for all economies and all agents. WR requires that the second order condition of payoff maximization holds strictly for a certain economy and, at least, for one agent. A motivation for this property is that it implies that, for this agent, to tell the truth is the unique best dominant strategy so she does have incentives not to cheat about her type.

Let $\hat{\theta}$ be an economy such that $\left(0, \omega_{1}, \omega_{2}, \ldots, \omega_{I}\right)$ is PE and there is, at least, one agent with nonlinear indifference curves.

Proposition 2. There is no DSIC and IR SCF defined on an open set $\Theta$ with $\hat{\theta} \in \Theta$ that is WR and PE at $\hat{\theta}$.

Proof. We first see that if $f(\hat{\theta})$ is a PE allocation, it must be that $f(\hat{\theta})=$ $\left(0, \omega_{1}, \omega_{2}, \ldots, \omega_{I}\right)$. Notice that any other PE allocation is such that either some agents have less utility than in $\left(0, \omega_{1}, \omega_{2}, \ldots, \omega_{I}\right)$, which contradicts that $f$ is IR or such that all agents are indifferent. In the latter case, a convex combination of this allocation and $\left(0, \omega_{1}, \omega_{2}, \ldots, \omega_{I}\right)$ can improve the utility of, at least, one individual, contradicting that $\left(0, \omega_{1}, \omega_{2}, \ldots, \omega_{I}\right)$ is PE.

Since $f$ is DSIC for all $\theta \in \Theta$, differentiating (12) we have that

$$
\frac{\partial \Omega\left(\theta, \tilde{\theta}_{i}\right)}{\partial \theta_{i}}+\frac{\partial \Omega\left(\theta, \tilde{\theta}_{i}\right)}{\partial \tilde{\theta}_{i}}=0 \text { for } \theta_{i}=\tilde{\theta}_{i} .
$$

Since $f$ is WR at $\hat{\theta}$ we have that for $\tilde{\theta}_{i}=\hat{\theta}_{i}, \frac{\partial \Omega\left(\hat{\theta}, \tilde{\theta}_{i}\right)}{\partial \theta_{i}}<0$ and thus,

$$
\frac{\partial \Omega\left(\hat{\theta}, \tilde{\theta}_{i}\right)}{\partial \tilde{\theta}_{i}} \equiv \frac{\partial^{2} U_{i}\left(y(\hat{\theta}), x_{i}(\hat{\theta}), \tilde{\theta}_{i}\right)}{\partial y \tilde{\theta}_{i}} \frac{\partial y(\hat{\theta})}{\partial \theta_{i}}+\frac{\partial^{2} U_{i}\left(y(\hat{\theta}), x_{i}(\hat{\theta}), \tilde{\theta}_{i}\right)}{\partial x_{i} \tilde{\theta}_{i}} \frac{\partial x_{i}(\hat{\theta})}{\partial \theta_{i}}>0
$$

If $\frac{\partial x_{i}(\hat{\theta})}{\partial \theta_{i}}=0$, Equation (12) above implies that $\frac{\partial y(\hat{\theta})}{\partial \theta_{i}}=0$ and the previous equation cannot hold. Thus, $\frac{\partial x_{i}(\hat{\theta})}{\partial \theta_{i}} \neq 0$. Therefore, there is a $\theta_{i}^{\prime}$ in a neighborhood of $\hat{\theta}_{i}$ such that $x_{i}\left(\theta_{i}^{\prime}, \hat{\theta}_{-i}\right)>x_{i}(\hat{\theta})=\omega_{i}$. Let,

$$
f\left(\theta_{i}^{\prime}, \hat{\theta}_{-i}\right)=\left(y\left(\theta_{i}^{\prime}, \hat{\theta}_{-i}\right), x_{1}\left(\theta_{i}^{\prime}, \hat{\theta}_{-i}\right), x_{2}\left(\theta_{i}^{\prime}, \hat{\theta}_{-i}\right), \ldots, x_{I}\left(\theta_{i}^{\prime}, \hat{\theta}_{-i}\right)\right) .
$$

Since $y\left(\theta_{i}^{\prime}, \hat{\theta}_{-i}\right) \geq 0$ and $x_{i}\left(\theta_{i}^{\prime}, \hat{\theta}_{-i}\right)>\omega_{i}$,

$$
U_{i}\left(y\left(\theta_{i}^{\prime}, \hat{\theta}_{-i}\right), x_{i}\left(\theta_{i}^{\prime}, \hat{\theta}_{-i}\right), \hat{\theta}_{i}\right)>U_{i}\left(0, \omega_{i}, \hat{\theta}_{i}\right)=U_{i}\left(f(\hat{\theta}), \hat{\theta}_{i}\right)
$$

Since $f$ is IR, $\forall j \neq i$, 


$$
U_{i}\left(y\left(\theta_{i}^{\prime}, \hat{\theta}_{-i}\right), x_{j}\left(\theta_{i}^{\prime}, \hat{\theta}_{-i}\right), \hat{\theta}_{j}\right) \geq U_{j}\left(0, \omega_{j}, \hat{\theta}_{j}\right)=U_{j}\left(f(\hat{\theta}), \hat{\theta}_{j}\right) .
$$

But this contradicts that $f$ is $\mathrm{PE}$ at $\theta=\hat{\theta}$.

Notice that, in contrast with other results in the literature, we only assume that $f$ selects PE allocations at $\theta=\hat{\theta}$. In particular, outside $\hat{\theta}$, the mechanism may pick up allocations where the budget balance equation (3) holds with strict inequality. We also notice that the result holds when the IR assumption is dropped and the SCF is required to be Envy-Free (a proof is available on request).

We now notice the following:

Remark 1. DSIC and WR imply that the single-crossing condition holds around $\hat{\theta}_{i}$ for agent $i$.

Proof: Notice that the FOC of DSIC can be written as

$$
\begin{gathered}
\frac{\partial U_{i}\left(y(\hat{\theta}), x_{i}(\hat{\theta}), \tilde{\theta}_{i}\right)}{\partial x_{i}}\left(M R S_{i}\left(\hat{\theta}, \tilde{\theta}_{i}\right) \frac{\partial y(\hat{\theta})}{\partial \theta_{i}}+\frac{\partial x_{i}(\hat{\theta})}{\partial \theta_{i}}\right) \equiv \Omega\left(\hat{\theta}, \tilde{\theta}_{i}\right)=0, \text { for } \theta_{i}=\tilde{\theta}_{i} . \\
\text { where } M R S_{i}\left(\hat{\theta}, \tilde{\theta}_{i}\right) \equiv \frac{\frac{\partial U_{i}\left(y(\hat{\theta}), x_{i}(\hat{\theta}), \tilde{\theta}_{i}\right)}{\partial y}}{\frac{\partial U_{i}\left(y(\hat{\theta}), x_{i}(\hat{\theta}), \tilde{\theta}_{i}\right)}{\partial x_{i}}} .
\end{gathered}
$$

Recall from the proof of Proposition 2 that DSIC and WR imply that $\frac{\partial \Omega\left(\hat{\theta}, \tilde{\theta}_{i}\right)}{\partial \tilde{\theta}_{i}}>$ 0 for $\tilde{\theta}_{i}=\hat{\theta}_{i}$. Thus,

$$
\begin{gathered}
\frac{\partial \Omega\left(\hat{\theta}, \tilde{\theta}_{i}\right)}{\partial \tilde{\theta}_{i}}=\frac{\partial^{2} U_{i}\left(y(\hat{\theta}), x_{i}(\hat{\theta}), \tilde{\theta}_{i}\right)}{\partial x_{i} \tilde{\theta}_{i}}\left(M R S_{i}\left(\hat{\theta}, \tilde{\theta}_{i}\right) \frac{\partial y(\hat{\theta})}{\partial \theta_{i}}+\frac{\partial x_{i}(\hat{\theta})}{\partial \theta_{i}}\right)+ \\
+\frac{\partial U_{i}\left(y(\hat{\theta}), x_{i}(\hat{\theta}), \tilde{\theta}_{i}\right)}{\partial x_{i}} \frac{\partial M R S_{i}\left(\hat{\theta}, \tilde{\theta}_{i}\right)}{\partial \tilde{\theta}_{i}} \frac{\partial y(\hat{\theta})}{\partial \theta_{i}}>0, \text { for } \tilde{\theta}_{i}=\hat{\theta}_{i}
\end{gathered}
$$

Notice that $\frac{\partial U_{i}\left(y(\hat{\theta}), x_{i}(\hat{\theta}), \tilde{\theta}_{i}\right)}{\partial x_{i}}>0$ implies that the FOC of DSIC is satisfied if and only if

$$
M R S_{i}\left(\hat{\theta}, \tilde{\theta}_{i}\right) \frac{\partial y(\hat{\theta})}{\partial \theta_{i}}+\frac{\partial x_{i}(\hat{\theta})}{\partial \theta_{i}}=0 .
$$

Then, from the inequality above,

$$
\frac{\partial U_{i}\left(y(\hat{\theta}), x_{i}(\hat{\theta}), \tilde{\theta}_{i}\right)}{\partial x_{i}} \frac{\partial M R S_{i}\left(\hat{\theta}, \tilde{\theta}_{i}\right)}{\partial \tilde{\theta}_{i}} \frac{\partial y(\hat{\theta})}{\partial \theta_{i}}>0
$$


and this implies $\frac{\partial M R S_{i}\left(\hat{\theta}, \tilde{\theta}_{i}\right)}{\partial x_{i}} \neq 0$, i.e. that the single-crossing condition holds around $\hat{\theta}_{i}$ for agent $i$.

Notice that a similar argument can be used to show that DSIC and R imply the single crossing assumption.

\section{Conclusions}

In this paper we have studied the incentives to tell the truth in domains that have as little structure as possible. We have obtained two main results: When there are two agents, there is no truth telling mechanism yielding efficient allocations and fulfilling A. 1. Recall that under this assumption, utility functions can be written as $H(y)+v_{i}\left(x_{i}, \theta_{i}\right)$. When the number of agents is arbitrary, there is no truth telling mechanism yielding individually rational allocations that is Weakly Regular and efficient at some economy of the domain.

Several questions remain open. 1) The use of the differential approach and, especially, the theory of differential equations to characterize DSIC mechanisms. 2) The use of the differential approach to study the performance of mechanisms when the equilibrium concept is Bayesian Equilibrium. 3) To prove our result on two-agents (i.e. Proposition 1) without using the additive separability assumption embedded in A. 1. All these are left for future research. 


\section{References}

[1] R. Bartle, The Elements of Real Analysis. Second edition, Wiley, NY, 1976.

[2] C. Beviá and L. C. Corchón, On the generic impossibility of truthful behavior, Econ.Theory 6, 1995, 365-71.

[3] D. E. Campbell and M. Truchon, Boundary optima and the theory of public good supply, J. Public Econ. 35, 1988, 241-9.

[4] E. Clarke, Multipart pricing of public goods, Public Choice 8, 1971, 19-33.

[5] J. Conley and D. Diamantaras, Generalized Samuelson conditions and welfare theorems for nonsmooth economies, J. Public Econ. 59, 1996, $137-52$.

[6] R. Deb and S. Ohseto, Strategy-proof and individually rational social choice functions for public good economies: A note, Economic Theory, 14, 1999, 685-689.

[7] T. Groves, Incentives in teams, Econometrica 41, 1973, 617-31.

[8] T. Groves and M. Loeb, Incentives and public inputs, J. Public Econ. 4, 1975, 211-26.

[9] J. Green and J. J. Laffont, Characterization of satisfactory mechanisms for the revelation of preferences for public goods, Econometrica 45, 1977, 427-438.

[10] J. Green and J. J. Laffont, Incentives in Public-Decision Making, North Holland, Amsterdam, 1979.

[11] B. Holmstrom, Groves' scheme on restricted domains, Econometrica 47, 1979, 1137-1144.

[12] L. Hurwicz and M. Walker, On the generic nonoptimality of dominantstrategy mechanisms, Econometrica 58, 1990, 683-704.

[13] C. Keser, Voluntary contributions to a public good when partial contribution is a dominant strategy, Econ. Letters 50, 1996, 359-66. 
[14] J. J. Laffont and E. Maskin, A differential approach to dominant strategy mechanisms, Econometrica 48 (1980), 1507-20.

[15] J. J. Laffont and E. Maskin, Nash and Dominant Strategy Implementation in Economic Environments, Journal of Mathematical Economics 10, 1982, 17-47.

[16] J. Ledyard and J. Roberts, On the incentive problem with public goods, Northwestern University Center for Mathematical Studies in Economics and Management Science, Discussion Paper 116, Northwestern University, 1975.

[17] H. Moulin, Serial cost sharing of excludable public goods, Rev. Econ. Stud. 61, 1994, 305-325.

[18] S. Ohseto, Strategy-proof mechanisms in public good economies, Mathematical Social Sciences 33, 1997, 157-183.

[19] B. Peleg, Double implementation of the Lindahl correspondence by a continuous mechanism, Econ. Design 2, 1996, 311-24.

[20] A. Postlewaite and D. Wettstein, Continuous and feasible implementation, Rev. Econ. Stud. 56, 1989, 603-11.

[21] T. Saijo, Incentive compatibility and individual rationality in public goods economies, J. Econ. Theory 55, 1991, 203-212.

[22] A. Saporiti, Strategy-proofness and single-crossing, Working Paper No. 49, 2007, Wallis Institute of Political Economy, University of Rochester.

[23] M. A. Satterthwaite and H. Sonnenschein, Strategy-proof allocation mechanisms at differentiable points, Rev. Econ. Stud. 53, 1981, 587597.

[24] J. Schummer, Strategy-proofness versus efficiency for small domains of preferences over public goods, Econ. Theory 13, 1999, 709-722.

[25] S. Serizawa, Strategy-Proof and Individually Rational Social Choice Functions for Public Good Economies, Economic Theory, 7, 1996, 501512. 
[26] S. Serizawa, Strategy-proof and individually rational social choice functions for public good economies, Econ. Theory 9, 1997, 379-380.

[27] S. Serizawa, Strategy-proof and symmetric social choice functions for public good economies, Econometrica 67, 1999, 121-145.

[28] S. Serizawa and J. Weymark, Efficient strategy-proof exchange and minimum consumption guarantees, Journal of Economic Theory 109, 2003, 246-263.

[29] G. Tian, On the existence of optimal truth-dominant mechanisms, Econ. Letters 53, 1996, 17-24.

[30] M. Walker, On the non-existence of dominant strategy mechanisms for making optimal public decisions Econometrica 48, 1980, 1521-40.

[31] E. C. Zachmanouglou and D. W. Thoe, Introduction to partial differential equations with applications, Dover, NY, 1986. 